\title{
Surgical Decision-Making in Spinal Instability in Facioscapulohumeral Muscular Dystrophy Related with a Spinal Muscle Atrophy
}

\author{
${ }^{1}$ Division of Spinal Surgery, IRCCS Fondazione Policlinico \\ Universitario Agostino Gemelli, Rome, Italy \\ ${ }^{2}$ Neurosurgery Division, Human Neurosciences Department, \\ "Sapienza" University, Italy \\ 3IRCCS "Neuromed", Pozzilli (IS), Italy \\ ${ }^{4}$ Division of Neurology, IRCCS Fondazione Policlinico Universitario \\ Agostino Gemelli, Rome, Italy \\ 5 Institute of Neurology, Università Cattolica del Sacro Cuore, Rome, \\ Italy \\ 6 Institute of Orthopaedic, Università Cattolica del Sacro Cuore, \\ Rome, Italy
}

Caterina Fumo ${ }^{1}$ Daniele Armocida ${ }^{2}$ Andrea Perna ${ }^{1}$ Alessandro Pesce ${ }^{3}$ Enzo Ricci ${ }^{4,5}$ Francesco Ciro Tamburrelli ${ }^{1,6}$ Alessandro Frati ${ }^{3}$ Antonio Santoro ${ }^{2}$ Luca Proietti ${ }^{1,6}$

J Neurosci Rural Pract 2021;12:445-446.

The indication and treatment of degenerative spinal pathology are specific to the preparation of the neurosurgeon specialist. However, there is a general lack of information on the possible surgical indication in patients suffering from rare genetic pathologies, such as myopathies, which can determine spinal deformities in adulthood. Facioscapulohumeral muscular dystrophy (FSHD) represents one of the most common genetic myopathies characterized by weakness of the facial and periscapular musculature ${ }^{1}$.

FSHD is characterized by the adipose infiltration of the face, shoulder, trunk, and limb muscles. ${ }^{2,3}$ While scapular and facial muscular degeneration is well-documented, few authors described the adipose degeneration that occurs in the paraspinal muscles of patients with FSHD, ${ }^{4}$ resulting in a pelvic imbalance that can cause different spinal deformities $^{3}$ like coronal and sagittal plane scoliosis and severe spondylolisthesis. Pure sagittal deformities such as lumbar hyperlordosis or flat back are relatively rare. ${ }^{5}$ In such a case, surgical correction is often considered. However, extensive traditional open spinal approaches may promote further trauma to the posterior spinal structures, and posterior spinal muscle, already impaired by atrophic degenerative changes. While several cases reported corrective surgical results on patients with Duchenne muscular dystrophy, cerebral palsy, and spinal muscular atrophy, few authors investigated the surgical results of milder but potentially inadequate, degenerative forms in terms of outcomes in

published online

March 24, 2021
DOI https://doi.org/

$10.1055 / \mathrm{s}-0041-1722816$ ISSN 0976-3147.
Address for correspondence Daniele Armocida, MD, AOU "Policlinico Umberto I," Viale del Policlinico 155, 00161 Roma, Italy (e-mail: danielearmocida@yahoo.it).

the case of FSHD patients. Minimally invasive spinal surgery, especially the extreme lateral interbody fusion (XLIF) approach, could minimize the surgical trauma, providing fair radiological and clinical outcomes, but was nevertheless never previously reported for the management of FSHD patients.

Whenever possible, a lateral fusion approach was combined with percutaneous pedicle screw fixation, ${ }^{4,5}$ which not only minimizes unnecessary trauma to the atrophic spinal muscles but also provides excellent radiological results and adequate indirect decompression. In our experience, surgically-treated FSHD patients showed improvement in posture and quality of life.

Before proceeding to a surgical procedure, a complete understanding of the etiology of the focal or diffuse deformities and the compensatory mechanism is mandatory. ${ }^{5}$ In the case of spinal muscle involvement in the natural history of a FSHD patient, spinal complications ranging from a single-level degenerative disc disease to a severe global spinal deformity can occur, and surgery should be seriously pondered for radiologically confirmed, progressively worsening spinal disorders, even in earlier stages, before deterioration of heart and lung function would possibly increase the probability of major postoperative complications.

\section{Conflict of Interest}

None declared.
(C) 2021. Association for Helping Neurosurgical Sick People. This is an open access article published by Thieme under the terms of the Creative Commons Attribution-NonDerivative-NonCommercial-License, permitting copying and reproduction so long as the original work is given appropriate credit. Contents may not be used for commercial purposes, or adapted, remixed, transformed or built upon. (https://creativecommons.org/licenses/by-nc-nd/4.0/). Thieme Medical and Scientific Publishers Pvt. Ltd. A-12, 2nd Floor, Sector 2, Noida-201301 UP, India 


\section{References}

1 Daher YH, Lonstein JE, Winter RB, Bradford DS. Spinal deformities in patients with muscular dystrophy other than Duchenne. A review of 11 patients having surgical treatment. Spine 1985;10(7):614-617

2 DeSimone AM, Pakula A, Lek A. Emerson CP Jr. Facioscapulohumeral muscular dystrophy. Compr Physiol 2017;7(4): $1229-1279$

3 Lee CS, Kang SJ, Hwang CJ, et al. Early-onset facioscapulohumeral muscular dystrophy - significance of pelvic extensors in sagittal spinal imbalance. J Pediatr Orthop B 2009; 18(6):325-329
4 Ricci E, Galluzzi G, Deidda G, et al. Progress in the molecular diagnosis of facioscapulohumeral muscular dystrophy and correlation between the number of KpnI repeats at the 4q35 locus and clinical phenotype. Ann Neurol 1999;45(6):751-757

5 Rijken NHM, van Engelen BGM, de Rooy JWJ, Geurts $\mathrm{ACH}$, Weerdesteyn V. Trunk muscle involvement is most critical for the loss of balance control in patients with facioscapulohumeral muscular dystrophy. Clin Biomech (Bristol, Avon) 2014;29(8):855-860 